\title{
Blood Amyloid Beta Levels in Healthy, Mild Cognitive Impairment and Alzheimer's Disease Individuals: Replication of Diastolic Blood Pressure Correlations and Analysis of Critical Covariates
}

\author{
Agustín Ruiz ${ }^{1 *}$, Pedro Pesini², Ana Espinosa ${ }^{1}$, Virginia Pérez-Grijalba ${ }^{2}$, Sergi Valero ${ }^{1,3}$, Oscar Sotolongo- \\ Grau ${ }^{1}$, Montserrat Alegret ${ }^{1}$, Inmaculada Monleón ${ }^{2}$, Asunción Lafuente ${ }^{1}$, Mar Buendía ${ }^{1}$, Marta Ibarria ${ }^{1}$, \\ Susana Ruiz¹, Isabel Hernández ${ }^{1}$, Itziar San José ${ }^{2}$, Lluís Tárraga ${ }^{1}$, Mercè Boada ${ }^{1,4}$, Manuel Sarasa ${ }^{2}$
}

1 Alzheimer Research Center and Memory Clinic, Fundació ACE, Institut Català de Neurociències Aplicades, Barcelona, Spain, 2 Araclon Biotech Ltd, Zaragoza, Spain, 3 Department of Psychiatry, Centro de Investigación Biomédica en Red en el Área de Salud Mental, Hospital Universitari Vall d'Hebron, Universitat Autònoma de Barcelona, Barcelona, Spain, 4 Hospital Universitari Vall d'Hebron, Institut de Recerca, Universitat Autònoma de Barcelona, Barcelona, Spain

\begin{abstract}
Plasma amyloid beta $(A \beta)$ levels are being investigated as potential biomarkers for Alzheimer's disease. In $A B 128$ cross-sectional study, a number of medical relevant correlates of blood A $\beta 40$ or A $\beta 42$ were analyzed in 140 subjects (51 Alzheimer's disease patients, 53 healthy controls and 36 individuals diagnosed with mild cognitive impairment). We determined the association between multiple variables with $A \beta 40$ and $A \beta 42$ levels measured in three different blood compartments called i) $A \beta$ directly accessible (DA) in the plasma, ii) $A \beta$ recovered from the plasma matrix (RP) after diluting the plasma sample in a formulated buffer, and iii) associated with the remaining cellular pellet (CP). We confirmed that diastolic blood pressure (DBP) is consistently correlated with blood DA A 340 levels ( $r=-0.19$, $\mathrm{P}=0.032$ ). These results were consistent in the three phenotypic groups studied. Importantly, the observation resisted covariation with age, gender or creatinine levels. Observed effect size and direction of AB40 levels/DBP correlation are in accordance with previous reports. Of note, DA A 40 and the RP A 40 were also strongly associated with creatinine levels $(r=0.599, P<<0.001)$ and to a lesser extent to urea, age, hematocrit, uric acid and homocysteine $(p<0.001)$. DBP and the rest of statistical significant correlates identified should be considered as potential confounder factors in studies investigating blood $A \beta$ levels as potential $A D$ biomarker. Remarkably, the factors affecting $A \beta$ levels in plasma (DA, RP) and blood cell compartments (CP) seem completely different.
\end{abstract}

Citation: Ruiz A, Pesini P, Espinosa A, Pérez-Grijalba V, Valero S, et al. (2013) Blood Amyloid Beta Levels in Healthy, Mild Cognitive Impairment and Alzheimer's Disease Individuals: Replication of Diastolic Blood Pressure Correlations and Analysis of Critical Covariates. PLoS ONE 8(11): e81334. doi: 10.1371/journal.pone.0081334

Editor: Maria Eugenia Saez, CAEBi, Spain

Received July 30, 2013; Accepted October 14, 2013; Published November 27, 2013

Copyright: @ 2013 Ruiz et al. This is an open-access article distributed under the terms of the Creative Commons Attribution License, which permits unrestricted use, distribution, and reproduction in any medium, provided the original author and source are credited.

Funding: This work has been funded by Araclon Biotech Ltd., Fundació ACE, and is also supported by the Spanish Ministry of Health from Instituto de Salud Carlos III (Madrid) (FISS PI10/00954) and by Agència d'Avaluació de Tecnologia i Recerca Mèdiques. Departament de Salut de la Generalitat de Catalunya (grant num. 390). The funder, Araclon Biotech Ltd., had a role in study design and data collection, but had no role on analysis, decision to publish, or preparation of the manuscript. The rest of funders had no role in study design, data collection and analysis, decision to publish, or preparation of the manuscript.

Competing interests: The authors have read the journal's policy and have the following conflicts: Agustín Ruiz is a shareholder of Neopharm Obesity SL and Oxigene Inc. Pedro Pesini, Virginia Pérez Grijalba, Inmaculada Monleón, Itziar San José, and Manuel Sarasa are employees of Araclon Biotech Ltd. Manuel Sarasa and Itziar San José are shareholders of Araclon Biotech Ltd. Mercè Boada is consultant to Novartis and Esteve pharmaceuticals. She is supported in part by funds from FIS/EC11-358 and FIS/P: 10-00945 of Ministerio de Sanidad, Servicios Sociales e Igualdad. Spain (AATM/390-6-2009) Generalitat de Catalunya (Catalan Government). She is a member of Advisory Boards of Grifols, Lilly, Elan, Nutricia, Genentech and Roche. Isabel Hernández, Ana Espinosa, Sergi Valero, Oscar Sotolongo-Grau, Montserrat Alegret, Asunción Lafuente, Mar Buendía, Marta Ibarria, Susana Ruiz and Lluís Tárraga have nothing to disclose. These declared conflicts do not alter the authors' adherence to all the PLOS ONE policies on sharing data and materials.

*E-mail: aruiz@fundacioace.com

\section{Introduction}

Alzheimer's disease (AD) is a global health problem for western countries, representing more than $60 \%$ of dementia cases in the world. The pathological findings of $A D$ include the progressive increase of $A \beta$ peptides in the brain conforming extracellular amyloid plaques together with intracellular deposits of hyper-phosphorylated tau that form characteristic 
neurofibrillary tangles. Both pathology hallmarks accompany progressive neuronal loss that ultimately provokes memory loss and severe cognitive dysfunction [1].

$A D$ is an intractable condition to date. The identification of early (preferably pre-symptomatic) biomarkers and true etiologic factors for this condition are the first steps to establish effective primary prevention programs for AD. Consequently, the search for a relatively inexpensive and harmless biomarker for $A D$ continues. Beyond the neuropsychological assessment which still represents the most essential tool for $A D$ and mild cognitive impairment $(\mathrm{MCl})$ screening in humans[2], the most reputed biomarkers for $A D$ are cerebral-spinal fluid (CSF) $A \beta 42$ and phosphorylated-tau protein levels, hippocampal volume measured by magnetic resonance imaging (MRI) techniques and positron electronic tomography (PET) scan with brain $A \beta$ radiotracers. These techniques represent the most studied methods for the detection of prodromal AD. However, there are also drawbacks for each one. For example, although MRI sensitivity showed high sensitivity at baseline, MRI specificity is not unexpectedly limited for $\mathrm{MCl}$ conversion to dementia[3] . Furthermore, MRI is restricted to patients with pacemakers. On the other hand, CSF measurements are sometimes variable and imprecise, since measurements vary between studies and laboratories, standardization of analytical as well as preanalytical procedures will be essential[4]. Some subjects may be unwilling to undergo a lumbar puncture or may have contraindications, such as use of anticoagulants. Finally, something that must be considered is the expense associated with performing amyloid PET scans in large numbers of subjects. Furthermore, its sensitivity and specificity for $\mathrm{MCl}$ conversion would require further evaluation, due to a need for improved clinical diagnosis of those subjects with major risk of conversion to dementia, and standardized protocols of data acquisition and imaging analyses. Therefore, opportunities for diagnosis improvement in prodromal or even pre-symptomatic $A D$ still remain.

In spite of an intensive worldwide research, there is not a definitive plasma or blood biomarker indicating high/low risk of conversion to Alzheimer's disease to date[5]. Because of their involvement in the generation of amyloid deposits in the brain, the $A \beta$ levels in blood have been widely investigated as potential markers for AD. However, regarding plasma measurements, contradicting results have been reached by using different molecular detection methods or research designs[6]. Beyond the role of plasma $A \beta$ levels as potential $A D$ biomarkers, there are a number of publications suggesting an association between plasma $A \beta$ levels and blood pressure[7-9], body mass index (BMI)[10-12], and different biochemical blood parameters such as insulin levels[13], creatinine [14-18], cystatin C[18,19] or homocystein levels $[15,16]$. These last observations are of importance because they might help understand the true physiological meaning of APP derived peptides in different tissues.

Our group is actively involved in the development of novel ELISA sandwich colorimetric tests for detection of $A \beta$ using whole blood instead of plasma alone [20,21]. In fact, using novel technology, we conducted a trial, called the $A B 128$ project, which studied the efficacy of $A \beta$ blood levels as an $A D$ biomarker. Specifically, we found statistical significant differences of some measurements in different blood compartments when comparing healthy controls $(\mathrm{HC})$ and $\mathrm{MCl}$ subjects [20]. In spite of these achievements, a definitive conclusion will require independent and extensive replication. Following this idea, a novel trial doubling sample size, the AB255 project, and independent replication efforts using samples obtained from reputed cross-sectional studies are underway to corroborate previous findings.

The aim of the present work is to identify the medical relevant variables correlated with blood $A \beta$ measurements obtained using these novel ELISA techniques. So, we have conducted an unsupervised search of such correlates in the available dataset obtained from the AB128 project. Our study confirmed several variables previously associated with plasma levels of $A \beta 40$ and pointed to others that seem specific for the amyloid retained in the cell pellet. The information provided herewith can help to define the critical set of covariates for future studies and might provide novel insights in the peripheral function of $A \beta$.

\section{Results}

First, we analyzed common demographics and clinical differences between the three phenotypic groups $(\mathrm{HC}, \mathrm{MCl}$ and AD) included in this study (for details see Methods section and table 1). Demographic analysis revealed important differences in age, education, plasma homocysteine levels and $A P O E$ allele $\varepsilon 4$ carrier status (dominant model) (Bonferroni corrected $\mathrm{p}<0.002$ ). However, no significant differences on gender, body mass index, creatinine plasma levels, heart rate, systolic blood pressure, diastolic blood pressure antihypertensive or statin treatment usage was observed. Strong differences on DA and RP A $\beta$ levels were also detected among groups $(p<0.001)$ as observed in a previous report[20].

Having these findings, we decided to start the covariate search for blood $A \beta$ by applying unsupervised searches in all phenotypic groups together (table S1a,b). Our study indicated that age, creatinine plasma levels and homocysteine were significantly correlated with DA and RP A 40 levels but not with $C P A \beta 40$ or $A \beta 42$ in any blood compartment (table 2). The study also suggested a significant, although not high, correlation between hematocrit, uric acid and diastolic blood pressure to different blood $A \beta$ measurements in blood. Interestingly, only serum levels of immunoglobulin $A$ can be nominally correlated to blood beta- $A \beta$ measures in cell pellet (CP) (table 2).

Previous studies have suggested a strong correlation between age and $A \beta$ levels, as well as between creatinine levels and $A \beta$ levels $[14,17,18]$. Other studies also indicated sexual dimorphism in amyloid measurements between men and women[17]. The demographic analysis suggested differences in age, $A P O E$ genotype and education among phenotypic groups. Moreover, the co-linearity between many parameters during unsupervised analysis was also observed, especially true for creatinine, homocysteine, uric acid and urea plasma levels (data not shown). Considering these observations, we decided to conduct a partial correlation 
Table 1. Demographics and clinical characteristics of subjects studied.

\begin{tabular}{|c|c|c|c|}
\hline Variable & Healthy Control & $\mathrm{MCl}$ & AD \\
\hline No. Of subjects & 53 & 36 & 51 \\
\hline Age $^{*}$, years & $60.3(8.3)$ & $75.1(6.4)$ & $78.3(5.7)$ \\
\hline Education* $(\%,>8$ years $)$ & 92.5 & 30.6 & 35.3 \\
\hline Gender (\% males) & 34 & 25 & 31.4 \\
\hline$A P O E^{*}$ (\% ع4 allele) & 18.9 & 52.8 & 64.7 \\
\hline Creatinine (mg/dl) & $0.76(.11)$ & $0.83(.19)$ & $0.86(.21)$ \\
\hline Homocysteine $^{*}(\mu \mathrm{mol} / \mathrm{L})$ & $9(3.7)$ & $13(4.2)$ & $13.5(3.9)$ \\
\hline Body Mass Index (BMI, kg/m²) & $26.6(4)$ & $26.9(3.7)$ & $26.7(4)$ \\
\hline Heart Rate (1/min) & $70.1(10)$ & $70.4(11)$ & $69.4(9)$ \\
\hline Systolic blood pressure (mmHg) & $134.5(21)$ & 144.6(18) & $144.5(21)$ \\
\hline Diastolic blood pressure $(\mathrm{mmHg})$ & $79.6(10)$ & 77.8(9) & $76.5(10)$ \\
\hline Hypertension treatment (\%) & 18.9 & 27.8 & 54.9 \\
\hline Statins treatment $(\%)$ & 10.8 & 19.4 & 27.5 \\
\hline $\mathrm{DA}$ a $\beta-40^{*}(\mathrm{pg} / \mathrm{ml})$ & $44.4(14)$ & $58.9(16)$ & $51(16)$ \\
\hline DA aß-42 (pg/ml) & $13(12)$ & $14(18)$ & 10.8(7.5) \\
\hline $\mathrm{RP}$ a $\beta-40^{*}(\mathrm{pg} / \mathrm{ml})$ & $84.5(16)$ & 95.5(18.9) & $95.5(18.1)$ \\
\hline $\mathrm{RP}$ a $\beta-42(\mathrm{pg} / \mathrm{ml})$ & $46.5(28)$ & $54.0(46)$ & $51.5(25)$ \\
\hline $\mathrm{CP}$ a $\beta-40(\mathrm{pg} / \mathrm{ml})$ & $59.4(9.6)$ & $55.2(13.5)$ & $60.8(11.3)$ \\
\hline $\mathrm{CP}$ aß-42 (pg/ml) & 149.1(69) & $151.9(70)$ & $165.2(67.9)$ \\
\hline Total amyloid in blood (pg/ml) & $339.7(90)$ & $356.7(101)$ & $373(78.2)$ \\
\hline \multicolumn{4}{|c|}{$\begin{array}{l}\text { For continuous variables the reported quantities are mean values with standard } \\
\text { deviations in brackets. }\end{array}$} \\
\hline \multicolumn{4}{|c|}{ *. P-value $<0.002$ (Bonferroni Corrected statistical significant threshold) } \\
\hline \multicolumn{4}{|l|}{ doi: 10.1371 /journal.pone.0081334.t001 } \\
\hline
\end{tabular}

analysis of candidate covariates, using an adjusted model including age, gender, phenotypic group, and creatinine levels (table 3). By applying this relatively simple model, we found that most of the correlates between blood $A \beta$ determinations and the other analyzed parameters disappeared, with the exception of DBP which maintained its correlation with RP $A \beta$ $40(r=-0.18 ; p=0.046 ;$ table 3$)$. Remarkably, an improved correlation between DBP and DA A 340 levels was observed using partial correlations analysis. This specific observation reached a nominally significant association $(r=-0.19 ; p=0.034$; table 3). Also, hematocrit correlations with RP A 40 ( $r=-0.29$; $p=0.001)$ and $D A A \beta 40(r=-0.23 ; p=0.01)$ remains.

These results pointed out that in addition to age, gender, creatinine, phenotype, the hematocrit and DBP could be interesting covariates for DA or RP blood $A \beta 40$ levels. In contrast, neither of them seemed important for blood $A \beta 42$ determinations (DA, RP or CP fractions) nor CP A $\beta 40$ (table 3).

Because age, anti-hypertensive drug usage and individual renal function differences among groups may severely distort DBP/DA A $\beta 40$, or DBP/RP A 40 relationships, we decided to conduct backward linear regression analyses in the three separate phenotypic groups (AD/MCl/HC). The main objective of this supplemental study was to ascertain whether there is a homogeneous correlation between DBP and DA A $\beta$ in plasma (in terms of effect size and direction) in separate groups. Figure 1 shows linear regression between diastolic blood pressure (DBP) volume and levels of DA $A B 40$ in the three groups separately. The analysis also permitted to check whether or not $D A A \beta 40$ can be retained in best regression models after extensive co-variation with well recognized parameters affecting DBP in elderly subjects (i.e. anti-hypertensive drug usage, BMI, age, gender, renal function variables, APOE genotype, etc.). Importantly, our results suggested a consistent correlation effect of DA A 40 levels in three phenotypic groups (table 4). Most importantly, in spite of including an extensive number of covariates, DA $A \beta 40$ was retained in the best backward regression model of the three phenotypic groups. This last result supports the notion that plasma $A \beta 40$ levels/DBP correlation was independent of well-established physiological and pathological parameters affecting blood pressure in elderly subjects. Furthermore, the association between both variables was important enough to be selected as a critical one irrespective of cognitive status of studied individuals (table 4).

Backward linear regression analyses also suggested that variables retained in the best model (and significantly affecting DBP) in the three phenotypic groups are different (table 4). In fact, cardiovascular factors such as total cholesterol levels and hematocrit seem much more important for DBP in the healthy (and younger) control group than in the $\mathrm{MCl}$ or $\mathrm{AD}$ groups. On the contrary, APOE genotype and creatinine levels seemed to only correlate well with the AD status. Remarkably, DA A 340 remains as one of the selected variables in the $\mathrm{MCl}$ group (table 4).

\section{Discussion}

The discovery of easily accessible, cost-effective and presymptomatic biomarkers for $A D$ is mandatory to fuel secondary prevention strategies. There are numerous efforts underway to reach this objective[5]. Technologies under investigation are multiple and not only related to amyloid levels. In fact, promising results are emerging by applying novel technologies such as analyses of differences in gene expression patterns[22,23], micro-RNA analyses [24] or proteomic-based approaches among others[25].

The efficacy of $A \beta$ blood levels on $A D$ prediction has also been recursively investigated. Original investigations suggested an association between $A \beta$ levels and $A D$, although results were not uniform among studies[6,8,9,19-21,26-28]. Thus, preliminary findings have remained controversial and would need independent validations $[6,19]$. Notably, the direct comparison between most studies is almost impossible due to the existence of many different immunodetection methods, including different polyclonal antibodies, differences on study designs or the lack of standardization or consensus in the analysis model. Therefore, the methods' differences in previously published studies prevent a direct comparison among currently available results or meta-analysis.

In the present study, the Araclon's blood $A \beta$ immunodetection system permitted the identification of previously observed well-established correlations, including blood pressure, hematocrit, creatinine and homocysteine levels. These observations are of importance mainly for two reasons: ( i) on the one hand, the identification of similar 
Table 2. Most significant correlations between beta-amyloid measurements and clinical parameters observed in this study.

\begin{tabular}{|c|c|c|c|c|c|c|c|c|}
\hline & & DA A $\beta 40$ & DA A $\beta 42$ & RP A $\mathbf{3 4 0}$ & RP A $\mathbf{3 4 2}$ & CP A $\beta 40$ & CP A 442 & Total amyloid \\
\hline AGE & Pearson's $r$ & $.370^{\star *}$ & .058 & $.341^{* \star}$ & .111 & .114 & .035 & .151 \\
\hline \multirow[t]{2}{*}{$(n=140)$} & p-value (2-tailed) & $6.73 \mathrm{E}-06$ & .495 & $3.74 \mathrm{E}-05$ & .192 & .181 & .682 & .074 \\
\hline & $\mathrm{Cl}(95 \%)$ & {$[0.208-0.512]$} & {$[-0.119-0.231]$} & [0.176 - 0.487] & {$[-0.066-0.281]$} & {$[-0.063-0.284]$} & {$[-0.210-0.142]$} & {$[-0.026-0.318]$} \\
\hline Creatinine (mg/dl) & Pearson's $r$ & $.599^{* *}$ & .153 & $.560^{\star *}$ & .112 & .087 & .025 & $.188^{*}$ \\
\hline \multirow[t]{2}{*}{$(n=124)$} & p-value (2-tailed) & $2.06 \mathrm{E}-13$ & .090 & $1.42 \mathrm{E}-11$ & .216 & .339 & .780 & .036 \\
\hline & $\mathrm{Cl}(95 \%)$ & {$[-0.473-0.701]$} & {$[-0.023-0.320]$} & {$[0.426-0.67]$} & {$[-0.050-0.292]$} & {$[-0.090-0.259]$} & {$[-0.151-0.200]$} & [0.013 - 0.352] \\
\hline DBP $(\mathrm{mmHg})$ & Pearson's $r$ & -.093 & -.102 & -.127 & $-.181^{*}$ & .013 & -.130 & $-.190^{*}$ \\
\hline \multirow[t]{2}{*}{$(n=140)$} & p-value (2-tailed) & .275 & .232 & .133 & .032 & .880 & .127 & .024 \\
\hline & $\mathrm{Cl}(95 \%)$ & {$[-0.264-0.084]$} & {$[-0.273-0.075]$} & {$[-0.296-0.050]$} & {$[-0.346-0.005]$} & {$[-0.136-0.188]$} & {$[-0.299-0.047]$} & {$[-0.354--0.015]$} \\
\hline Hematocrit (\%) & Pearson's $r$ & $-.305^{\star *}$ & -.022 & $-.288^{\star *}$ & -.104 & .044 & .124 & .002 \\
\hline \multirow[t]{2}{*}{$(n=124)$} & p-value (2-tailed) & .001 & .807 & .001 & .252 & .630 & .171 & 981 \\
\hline & $\mathrm{Cl}(95 \%)$ & {$[-0.456--0.136]$} & {$[-0.197-0.154]$} & {$[-0.441-0.118]$} & {$[-0.275-0.073]$} & {$[-0.133-0.218]$} & {$[-0.053-0.293]$} & {$[-0.178-0.228]$} \\
\hline Homocysteine ( $\mathrm{mcmol} / \mathrm{L})$ & Pearson's $r$ & $.325^{\star \star}$ & .069 & $.345^{\star \star}$ & .097 & .019 & .070 & .164 \\
\hline \multirow[t]{2}{*}{$(n=124)$} & p-value (2-tailed) & $2.28 \mathrm{E}-04$ & .444 & 8.63E-05 & .286 & .837 & .439 & .069 \\
\hline & $\mathrm{Cl}(95 \%)$ & {$[0.158-0.474]$} & {$[-0.242-0.108]$} & [0.18 - 0.491] & {$[-0.080-0.268]$} & {$[-0.157-0.194]$} & {$[-0.107-0.243]$} & {$[-0.012-0.330]$} \\
\hline $\begin{array}{l}\text { Serum Immunoglobulin A } \\
(\mathrm{mg} / \mathrm{dL})\end{array}$ & Pearson's $r$ & .130 & .149 & .063 & .125 & $.180^{*}$ & $.221^{*}$ & $.253^{* *}$ \\
\hline \multirow[t]{2}{*}{$(n=123)$} & p-value (2-tailed) & .149 & .099 & .485 & .166 & .045 & .014 & .005 \\
\hline & $\mathrm{Cl}(95 \%)$ & {$[-0.047-0.299]$} & {$[-0.028-0.316]$} & {$[-0.114-0.236]$} & {$[-0.052-0.294]$} & {$[0.004-0.345]$} & {$[0.047-0.382]$} & {$[0.081-0.41]$} \\
\hline Urea (mg/dl) & Pearson's $r$ & $.398^{* *}$ & .069 & $.319^{* *}$ & .074 & -.006 & -.012 & .083 \\
\hline \multirow[t]{2}{*}{$(n=124)$} & p-value (2-tailed) & 4.74E-06 & .447 & 3.11E-04 & .412 & .947 & .891 & .357 \\
\hline & $\mathrm{Cl}(95 \%)$ & {$[0.239-0.536]$} & {$[-0.242-0.108]$} & {$[0.152-0.468]$} & {$[-0.103-0.247]$} & {$[-0.182-0.170]$} & {$[-0.187-0.164]$} & {$[-0.094-0.255]$} \\
\hline Uric acid (mg/dl) & Pearson's r & $.285^{\star \star}$ & .149 & $.259^{\star \star}$ & .049 & .165 & .000 & .093 \\
\hline \multirow[t]{2}{*}{$(n=124)$} & p-value (2-tailed) & .001 & .099 & .004 & .591 & .067 & 1.000 & .304 \\
\hline & $\mathrm{Cl}(95 \%)$ & {$[0.115-0.439]$} & {$[-0.028-0.316]$} & {$[0.087-0.416]$} & {$[-0.128-0.223]$} & {$[-0.011-0.331]$} & {$[-0.176-0.176]$} & {$[-0.084-0.264]$} \\
\hline
\end{tabular}

${ }^{*} \mathrm{p}<0.05$ (2-tailed). ${ }^{* *} \mathrm{P}<.01$

doi: $10.1371 /$ journal.pone.0081334.t002

Table 3. Partial correlations between $A \beta$ measurements and clinical parameters observed in this study adjusted by Age, creatinine, gender and phenotype.

\begin{tabular}{|c|c|c|c|c|c|c|c|c|}
\hline & & DA A $\beta 40$ & DA A $\beta 42$ & RP A $\beta 40$ & RP A $\beta 42$ & CP A $\beta 40$ & CP A $\beta 42$ & Total amyloid \\
\hline DBP & Pearson's $r$ & -.195 & -.130 & -.183 & -.171 & -.045 & -.061 & -.148 \\
\hline \multirow[t]{2}{*}{$(n=124)$} & p-value (2-tailed) & .032 & 156 & .046 & .062 & .627 & .511 & 108 \\
\hline & $\mathrm{Cl}(95 \%)$ & {$[-0.358--0.020]$} & {$[-0.299--0.047]$} & {$[-0.348--0.007]$} & {$[-0.324-0.019]$} & {$[-0.219-0.132]$} & {$[-0.234-0.116]$} & {$[-0.316-0.029]$} \\
\hline Hematocrit (\%) & Correlation & -.288 & -.044 & -.231 & -.130 & .071 & .112 & .010 \\
\hline \multirow[t]{2}{*}{$(n=124)$} & p-value (2-tailed) & .001 & .631 & .011 & .157 & .442 & .225 & .910 \\
\hline & $\mathrm{Cl}(95 \%)$ & {$[-0.441--0.118]$} & {$[-0.218-0.133]$} & {$[-0.437--0.057]$} & {$[-0.299-0.103]$} & {$[-0.106-0.244]$} & {$[-0.065-0.282]$} & {$[-0.166-0.185]$} \\
\hline Homocysteine (mcmol/L) & Pearson's $r$ & -.049 & -.008 & .041 & .012 & -.069 & .054 & .044 \\
\hline \multirow[t]{2}{*}{$(n=124)$} & p-value (2-tailed) & .598 & .928 & .657 & .900 & .455 & .557 & .630 \\
\hline & $\mathrm{Cl}(95 \%)$ & {$[-0.223-0.128]$} & {$[-0.184-0.168]$} & {$[-0.136-0.215]$} & {$[-0.164-0.187]$} & {$[-0.242-0.108]$} & {$[-0.123-0.228]$} & {$[0.286-0.571]$} \\
\hline $\begin{array}{l}\text { Serum Immunoglobulin A } \\
(\mathrm{mg} / \mathrm{dL})\end{array}$ & Pearson's $r$ & .019 & .120 & -.044 & .081 & .158 & .207 & .207 \\
\hline \multirow[t]{2}{*}{$(n=123)$} & p-value (2-tailed) & .839 & 192 & .637 & .376 & .085 & .023 & .023 \\
\hline & $\mathrm{Cl}(95 \%)$ & {$[-0.194-0.157]$} & {$[-0.057-0.290]$} & {$[-0.218-0.133]$} & {$[-0.096-0.253]$} & {$[-0.018-0.325]$} & {$[0.032-0.369]$} & [0.032 - 0.369] \\
\hline Urea (mg/dl) & Pearson's r & .146 & -.012 & .059 & .004 & -.061 & -.032 & -.022 \\
\hline \multirow[t]{2}{*}{$(n=124)$} & p-value (2-tailed) & .111 & .894 & .519 & .969 & .508 & .728 & .809 \\
\hline & $\mathrm{Cl}(95 \%)$ & {$[-0.031-0.314]$} & {$[-0.187-0.164]$} & {$[-0.118-0.232]$} & {$[-0.172-0.180]$} & {$[-0.234-0.116]$} & {$[-0.207-0.145]$} & {$[-0.197-0.154]$} \\
\hline Uric Acid (mg/dl) & Pearson's r & -.074 & .037 & -.056 & -.056 & .160 & -.040 & -.040 \\
\hline \multirow[t]{2}{*}{$(n=124)$} & p-value (2-tailed) & .424 & .690 & .546 & .546 & .081 & 663 & .661 \\
\hline & $\mathrm{Cl}(95 \%)$ & {$[-0.247-0.103]$} & {$[-0.140-0.211]$} & {$[-0.230-0.121]$} & {$[-0.230-0.121]$} & {$[-0.016-0.327]$} & {$[-0.214-0.137]$} & {$[-0.214-0.137]$} \\
\hline
\end{tabular}


Table 4. Backward linear regression analysis exploring factors correlated with diastolic blood pressure.

\begin{tabular}{|c|c|c|c|c|c|c|c|}
\hline \multirow[b]{2}{*}{ Groups } & & \multicolumn{2}{|c|}{ Unstandardized Coefficients } & \multicolumn{2}{|l|}{ Standardized Coefficients } & \multirow{2}{*}{$\begin{array}{l}95.0 \% \text { Confidence Interval } \\
\text { for B } \\
\text { Lower Bound }\end{array}$} & \multirow{2}{*}{$\begin{array}{l}95.0 \% \text { Confidence Interval } \\
\text { for B } \\
\text { Upper Bound }\end{array}$} \\
\hline & & B & Std. Error & Beta & Sig. & & \\
\hline Group 1 & (Constant) & 60.755 & 6.090 & & .000 & 48.455 & 73.054 \\
\hline Model 9 & $\mathrm{DA} A \beta 40$ & -.261 & .092 & -.481 & .007 & -.447 & -.075 \\
\hline \multirow[t]{4}{*}{$A D$} & Creatinine & 23.416 & 7.282 & .559 & .003 & 8.710 & 38.121 \\
\hline & APOE & 5.124 & 2.411 & .280 & .040 & .254 & 9.994 \\
\hline & Statins & 4.060 & 2.494 & .211 & .111 & -.977 & 9.097 \\
\hline & Vitamin B12 & .010 & .006 & .241 & .071 & -.001 & .021 \\
\hline Group 2 & (Constant) & 38.873 & 15.731 & & .019 & 6.788 & 70.957 \\
\hline Model 10 & $\mathrm{DA} A \beta 40$ & -.318 & .139 & -.551 & .030 & -.602 & -.034 \\
\hline \multirow[t]{3}{*}{ MCl } & Creatinine & 30.277 & 13.790 & .627 & .036 & 2.152 & 58.401 \\
\hline & Sex & 7.632 & 4.365 & .363 & .090 & -1.269 & 16.534 \\
\hline & BMI & .708 & .387 & .290 & .077 & -.081 & 1.497 \\
\hline Group 3 & (Constant) & 4.741 & 16.357 & & .774 & -28.538 & 38.021 \\
\hline Model 11 & DA A 340 & -.233 & .126 & -.263 & .074 & -.489 & .024 \\
\hline \multirow[t]{2}{*}{ Healthy Controls } & Cholesterol & .169 & .049 & .468 & .002 & .069 & .270 \\
\hline & Hematocrit & 1.100 & .290 & .499 & .001 & .510 & 1.690 \\
\hline
\end{tabular}

doi: 10.1371/journal.pone.0081334.t004

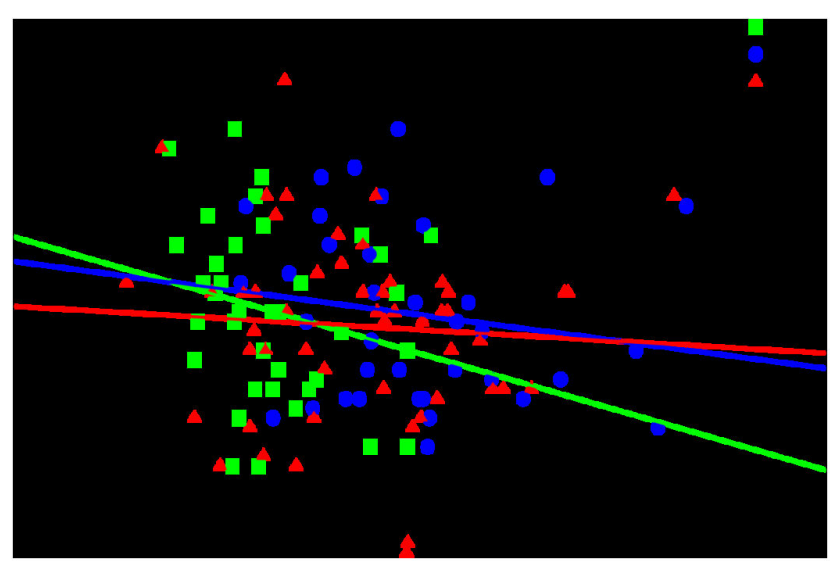

Figure 1. Linear regression between diastolic blood pressure (DBP) volume and levels of DA $A ß 1-40$ in Alzheimer's disease patients ( $A D$, red triangles), mild cognitive impairment subjects ( $\mathrm{MCl}$, blue circles) and healthy individuals (HC, green squares).

doi: 10.1371/journal.pone.0081334.g001

correlations to those previously reported by independent research groups using our technology directly reassured the validity of our methods; (ii) on the other hand, the identification of such covariates may serve to a better design of future larger studies by controlling critical covariates affecting $A \beta$ levels.

Based on previous investigations and on the findings presented here, variables such as age, gender and renal function biomarkers (i.e. serum creatinine levels) appeared as the most important covariates correlated to the levels of plasma (DA or RP) A $\beta$ 40. The effect of these covariates in the blood levels of $A \beta 42$ appear to be smaller in our study. However, taking into account previous results, the incorporation of such covariates to future analyses also seems pretty reasonable for studies with $A \beta 42$. This could seem like an apparently obvious recommendation. In fact, it has been previously suggested by others[14]. However, only a minority of studies such as the cardiovascular health study (CHS) has incorporated renal function biomarkers in their models [19].

In contrast, the rest of correlates initially detected seem not to be independent of creatinine levels. Thence, we suggest that urea, uric acid and homocysteine associations have appeared only as a consequence of strong creatinine/ $A \beta$ relationship as previously mentioned. Consequently, it makes no sense to incorporate them to regression models due to high co-linearity among them.

An exception to this rule might be the hematocrit, which retained its correlation with $A \beta 40$ levels even after the adjustments of age, gender, phenotype and plasmatic creatinine levels. It is possible that the hematocrit fraction is somehow affecting the equilibrium between cell-bound and cell-free in plasma amyloid. However, this association was not uniform enough among the phenotypic groups $(\mathrm{HC}, \mathrm{MCl}$ and $\mathrm{AD})$ to delineate a definitive conclusion. A replication increasing sample size is necessary to corroborate hematocrit findings. Interestingly, these results are consistent with previous findings in the Japanese population suggesting a genuine correlation between both physiological parameters[7]. Although some authors have speculated on hematocrit implication on cognition performance[29], its role on $A D$ etiology is not well understood and deserves further investigation.

On the contrary, blood pressure (BP) is a well-recognized risk factor for dementia. Indeed, $A D$ has been linked to cardiovascular risk factors, such as diabetes mellitus, hyperlipidemia, and, in particular, elevated BP[9]. DBP/A 340 correlations appear consistent in our study irrespective of the individual's phenotype. This observation corroborated, in terms 
of effect size and direction, previous reports $[8,30]$. The largest study reported to date on blood pressure and plasma $A \beta$ levels was held by Lambert et al. in their "Three-Cities Study"[8]. The authors suggested associations between the $A \beta 40 / A \beta 42$ ratio and the systolic blood pressure, the DBP or the hypertension. However, they also pointed out that the observed effect could be mainly driven by plasma $A \beta$ 40. Furthermore, the mechanism underlying this preferential association could be related to the $A \beta 40$ peptide's properties on vascular vessels (for details, see Lambert et al. and references therein). Concretely, previous studies have indicated that $A \beta 40$ peptides could modify cerebral blood vessels in vitro and induce a decrease of cerebral flow and cerebral blood volume in vivo[31,32] and that $A \beta 40$ has important effects on vasoconstriction[32]. Our results independently confirm the importance of $A \beta 40$ in blood pressure in humans. Most importantly, the stratified analysis suggests that this effect can be detected in elderly patients affected or unaffected by $\mathrm{MCl}$ and $A D$.

The relationships between blood $A \beta$ levels, blood pressure and Alzheimer's disease have also been explored in the Honolulu Heart Program/Honolulu Asia Aging Study[9]. The authors of that study suggested that the risk for $A D$ significantly increased with lower levels of plasma $A \beta$ 40; hazard ratio: 2.1 [95\% Cl: 1.4 to 3.1]; and detected evidence of interaction between DBP and plasma $A \beta 40$. Importantly, low plasma $A \beta$ 40 or 42 was associated with the presence of cerebral amyloid angiopathy but not with the other neuropathologies. Therefore, the disruption of blood pressure homeostasis in midlife could contribute to future risk of dementia. Therefore, reduced levels of $A \beta 40$ in midlife could be directly or indirectly involved in the early pathogenic process of $A D$.

A major limitation of this study is the observed differences in demographic characteristics among groups. In general, healthy controls are younger and with higher educational scores than $\mathrm{MCl}$ or AD subjects. However, despite this limitation the study was able to replicate previous findings as already pointed out, although the new insights shown here would deserve a further replication.

Another limitation would be the small sample size. This might explain the discordance observed in the relationships between amyloid measurements in different compartments and physiological parameters studied (Table 2 and 3 ). For example, backward regression analyses using $A \beta 40 \mathrm{RP}$ fraction versus DBP were not fully consistent with the results obtained in $A \beta 40$ DA compartment (data not shown). This divergence might be attributable to having a small sample size which in turn may provoke random chance oscillations during effect size estimation. Alternatively, unknown factors could be affecting peptide measurements in different compartments. For this reason, it would be advisable to independently replicate these results to corroborate these findings.

Finally, the biological meaning of these findings cannot be ignored because it could provide essential information about the real physiology of APP derived peptides in different human systems. For instance, the creatine/creatinine energy cycle in brain and muscle could have a closer relation with amyloid physiology than previously anticipated [33]. Therefore, observed correlations would need a more deep interpretation beyond a mere correlation with the renal function as proposed in previous studies. In contrast, the covariates related to $A \beta C P$ fraction remained almost unknown. In fact, only serum immunoglobulin A levels displayed a weak correlation with the amyloid measured in the cell pellet fraction of the blood. Importantly, the presence of immunoglobulin fragments in the amyloid plaques of the AD brain had been observed[34] and this observation could be easily linked with $A D$ neuroinflammatory hypothesis[35] . However, we considered that this last observation would require independent confirmation in future studies. Nevertheless, the novelty and potential of CP amyloid warrant further investigation.

\section{Materials and Methods}

\section{Patients}

We selected one hundred and forty subjects from the AB128 project. All these patients were recruited at a single clinical research site, the Memory Clinic of Fundació ACE, Institut Català de Neurociències Aplicades, Barcelona, Spain. The original design included three phenotypic groups of individuals: AD patients ( $n=51 ; 31.4 \%$ males), mild cognitive impairment (MCl) patients ( $n=36 ; 25 \%$ males) and healthy controls $(\mathrm{HC})$ $(n=53 ; 34 \%$ males). The demographic characteristics of the subjects under study are summarized in Table 1.

$\mathrm{AD}, \mathrm{HC}$ and $\mathrm{MCl}$ criteria used to recruit subjects in this study have been described in our previous works[20,21]. Cognitive assessment was performed according to the routines of the Memory Clinic of Fundació ACE as described elsewhere[36]. Briefly, $\mathrm{MCl}$ subjects fulfilled the Petersen's diagnostic criteria[37] including subjective memory complaint, normal general cognition, preserved performance in activities of daily living, absence of dementia and a measurable impairment in memory function, with or without deficit in other cognitive domains[38]. All $\mathrm{MCl}$ subjects had a CDR rating of 0.5. Based on the Wechsler Memory Scale-III (WMS-III) of NBACE battery an impaired delayed verbal recall for which recognition testing did not improve performance, classified $\mathrm{MCl}$ amnesic subjects as having an "Encoding/Storage" pattern of memory loss. Diagnosis of AD individuals followed NINCDS-ADRDA criteria[39,40], a CDR of 1 point or more and a Mini-Mental State Examination (MMSE) below 24 points. Healthy controls were cognitively normal when evaluated in the Fundació ACE, had MMSE scores of at least 26 (considering that the MMSE cut-off has been demonstrated to be $<25$ in the Spanish population [41]), and also had a normal neuroimaging MRI profile.

A written informed consent was obtained from every participant. The study's protocols were reviewed and approved by the Ethical Committee of the Hospital Clinic i Provincial (Barcelona, Spain).

\section{Blood sampling, biochemical determinations and $A \beta$ measurements}

Blood samples from each participant were routinely processed in Fundació ACE as previously described[21]. Plasma biochemical and hematologic measurements were 
obtained in a reference laboratory according to routine clinical standards. For blood amyloid testing, all samples were analyzed in triplicate in the same laboratory and blinded for analysts. For each of the three blood fractions analyzed, two specific ELISA sandwich kits, ABtest 40 and ABtest 42 (Araclon Biotech Ltd. Zaragoza, Spain) were used as described elsewhere. Briefly, before analysis, plasma and blood cell samples were pretreated by dilution in a formulated saline buffer with $1 \%$ blocking polymer according to the supplier's instructions. We determined three parameters for both the $A \beta 40$ and $A \beta 42$ peptides in each blood sample. One determination was performed using the undiluted plasma sample, another using the plasma sample diluted 1:3 with the aforementioned formulated buffer, and a third using the cellular pellet that remained after plasma collection. The peptide amount in the undiluted plasma sample corresponds to the directly accessible (DA) peptide. The 1:3 dilution of the plasma was chosen because it provided the maximum peptide recovery from the plasmatic sample. Thus, this determination included the DA peptide and the peptide that was recovered from the plasma matrix (RP). Additionally, the peptide associated with the cellular pellet (CP) was measured in a 1:5 dilution of the pellet that remained after plasma collection.

\section{Statistical Analysis}

a) Unsupervised Pearson's correlation analyses. $A \beta 40$ and $A \beta 42$ peptides measurements in three different blood compartments (DA, RP and CP), several calculated indices derived from these primary measurements and thirty seven medical relevant variables obtained from 140 individuals were sorted and arranged in a text file. Variable name, data source, units and main statistical characteristics of each variable are detailed in table 1 and table S1a and S1b.

To calculate standardized Pearson's coefficients, the constructed text file was processed using the $\mathrm{R}$ statistical package according to programmers' instructions[42]. $R$ command cor was selected for this purpose because it permits automatic (and appropriated) management of missing cells. Pearson's coefficients of determination $\left(r^{2}\right)$ were easily derived using $\mathrm{R}$ calculation tools (table $\mathrm{S} 1 \mathrm{~b}$ ).

Top correlated variables for each primary measurement plus total $A \beta$ in blood were filtered and ranked using conventional excel spreadsheets (table S1a,b). Selected candidate covariates for each primary measurement were chosen for further research.

b) Partial correlation and regression-based analyses. On the basis of Pearson's coefficients analysis, eight variables (i.e. creatinine $(\mathrm{mg} / \mathrm{dl})$, urea $(\mathrm{mg} / \mathrm{dl})$, age at baseline (years), homocysteine $(\mathrm{mcmol} / \mathrm{L})$, uric acid $(\mathrm{mg} / \mathrm{dl})$, serum immunoglobulin A $(\mathrm{mg} / \mathrm{dL})$, diastolic blood pressure (DBP) $(\mathrm{mmHg})$ and Hematocrit (\%) were selected for further research. We used SPSS 18 package (PASW Statistics for Windows,
Version 18.0. Chicago: SPSS Inc.) to re-calculate Pearson's coefficient of determination $\left(r^{2}\right)$ and statistical significance of all selected variables and blood $A \beta$ measurements. Partial correlation analyses were conducted to calculate adjusted correlation coefficients of each selected variable and $A \beta$ levels. Adjusted covariables for partial correlation analyses used were age, gender, phenotypic group $(\mathrm{AD} / \mathrm{MCl} / \mathrm{HC})$ and plasmatic creatinine.

To further demonstrate the independence of DBP/ $A \beta$ relationships with the rest of co-variables or phenotypic status, we conducted a backward regression analysis on each phenotypic group separately. In this specific analysis the choice of predictive variables was carried out by an automatic procedure using SPSS 18 on each phenotypic group. Backward elimination of variables, which involves starting with multiple variables affecting DBP (DA A $\beta$ 40, creatinine, APOE genotype, gender, age, body mass index, antihypertensive treatment, statins treatment, cholesterol levels, triglycerides level, vitamin b12 levels, hematocrit and homocysteine), testing the deletion of each variable using a chosen model comparison criterion, deleting the variable (if any) that improves the model the most by being deleted, and repeating this process until no further improvement is possible. Regression-based analyses of $\mathrm{PAD} / \mathrm{A} \beta$ were graphically represented using gnuplot 4.6 (URL http://gnuplot.info).

\section{Supporting Information}

Table S1. A: Pearson coefficients ( $r$ ) between plasma amyloid levels and medical variables. B: Pearson coefficients $\left(r^{2}\right)$ between plasma amyloid levels and medical variables.

(XLSX)

\section{Acknowledgements}

We would like to thank patients and controls who participated in this project. We are indebted to Trinitat Port-Carbó and her family who are supporting Fundació ACE research programs. Fundació ACE is an associated center to CIBERNED. Anonymised data table containing this dataset is available on request to corresponding author.

\section{Author Contributions}

Conceived and designed the experiments: MS M. Boada LT ISJ PP AR SV OS. Performed the experiments: PP AE VPG MA IM AL M. Buendía MI SR IH. Analyzed the data: AR SV OS PP M. Boada LT MS. Contributed reagents/materials/analysis tools: PP VPG IM SV OS AR. Wrote the manuscript: AR OS PP AE MS M. Boada LT. 


\section{References}

1. Ballard C, Gauthier S, Corbett A, Brayne C, Aarsland D et al. (2011) Alzheimer's disease. Lancet 377: 1019-1031. doi:10.1016/ S0140-6736(10)61349-9. PubMed: 21371747.

2. Soininen HS, Scheltens $P$ (1998) Early diagnostic indices for the prevention of Alzheimer's disease. Ann Med 30: 553-559. doi: 10.3109/07853899809002604. PubMed: 9920358

3. Davatzikos C, Bhatt $P$, Shaw LM, Batmanghelich KN, Trojanowski JQ (2011) Prediction of $\mathrm{MCl}$ to AD conversion, via MRI, CSF biomarkers, and pattern classification. Neurobiol Aging 32: 2322: e2319-2327. PubMed: 20594615.

4. Rosén C, Hansson O, Blennow K, Zetterberg H (2013) Fluid biomarkers in Alzheimer's disease - current concepts. Mol Neurodegener 8: 20. doi:10.1186/1750-1326-8-S1-P20. PubMed: 23800368.

5. Fletcher LC, Burke KE, Caine PL, Rinne NL, Braniff CA et al. (2013) Diagnosing Alzheimer's disease: are we any nearer to useful biomarker-based, non-invasive tests? GMS health technology assessment 9: Doc01.

6. Toledo JB, Shaw LM, Trojanowski JQ (2013) Plasma amyloid beta measurements - a desired but elusive Alzheimer's disease biomarker. Alzheimers Res Ther 5: 8. doi:10.1186/alzrt162. PubMed: 23470128.

7. Fujiwara Y, Takahashi M, Tanaka M, Hoshi T, Someya T et al. (2003) Relationships between plasma beta-amyloid peptide 1-42 and atherosclerotic risk factors in community-based older populations. Gerontology 49: 374-379. doi:10.1159/000073765. PubMed: 14624066.

8. Lambert JC, Dallongeville J, Ellis KA, Schraen-Maschke S, Lui J et al. (2011) Association of plasma Ass peptides with blood pressure in the elderly. PLOS ONE 6: e18536. doi:10.1371/journal.pone.0018536. PubMed: 21525986

9. Shah NS, Vidal JS, Masaki K, Petrovitch H, Ross GW et al. (2012) Midlife blood pressure, plasma beta-amyloid, and the risk for Alzheimer disease: the Honolulu Asia Aging Study. Hypertension 59: 780-786. doi:10.1161/HYPERTENSIONAHA.111.178962. PubMed: 22392902.

10. Balakrishnan K, Verdile G, Mehta PD, Beilby J, Nolan D et al. (2005) Plasma Abeta42 correlates positively with increased body fat in healthy individuals. J Alzheimers Dis 8: 269-282. PubMed: 16340084.

11. Toledo JB, Toledo E, Weiner MW, Jack CR Jr., Jagust W et al. (2012) Cardiovascular risk factors, cortisol, and amyloid-beta deposition in Alzheimer's Disease Neuroimaging Initiative. Alzheimer'S and Dementia : the Journal of the Alzheimer'S Association 8: 483-489. doi: 10.1016/j.jalz.2011.08.008

12. Vidoni ED, Townley RA, Honea RA, Burns JM (2011) Alzheimer disease biomarkers are associated with body mass index. Neurology 77: 1913-1920. doi:10.1212/WNL.0b013e318238eec1. PubMed: 22105948

13. Townsend MK, Okereke OI, Xia W, Yang T, Selkoe DJ et al. (2012) Relation between insulin, insulin-related factors, and plasma amyloid beta peptide levels at midlife in a population-based study. Alzheimer Dis Assoc Disord 26: 50-54. doi:10.1097/WAD.0b013e31821764ce. PubMed: 21502851

14. Arvanitakis Z, Lucas JA, Younkin LH, Younkin SG, Graff-Radford NR (2002) Serum creatinine levels correlate with plasma amyloid Beta protein. Alzheimer Dis Assoc Disord 16: 187-190. doi: 10.1097/00002093-200207000-00009. PubMed: 12218650.

15. Irizarry MC, Gurol ME, Raju S, Diaz-Arrastia R, Locascio JJ et al. (2005) Association of homocysteine with plasma amyloid beta protein in aging and neurodegenerative disease. Neurology 65: 1402-1408. doi:10.1212/01.wnl.0000183063.99107.5c. PubMed: 16275827

16. Luchsinger JA, Tang MX, Miller J, Green R, Mehta PD et al. (2007) Relation of plasma homocysteine to plasma amyloid beta levels. Neurochem Res 32: 775-781. doi:10.1007/s11064-006-9207-7. PubMed: 17191133.

17. Metti AL, Cauley JA, Ayonayon HN, Harris TB, Rosano C et al. (2012) The Demographic and Medical Correlates of Plasma Abeta40 and Abeta42. Alzheimer Disease and Associated Disorders.

18. Rajagopalan P, Refsum H, Hua X, Toga AW, Jack CR Jr. et al. (2013) Mapping creatinine- and cystatin C-related white matter brain deficits in the elderly. Neurobiol Aging 34: 1221-1230. doi:10.1016/ j.neurobiolaging.2012.10.022. PubMed: 23182131.

19. Lopez OL, Kuller LH, Mehta PD, Becker JT, Gach HM et al. (2008) Plasma amyloid levels and the risk of $A D$ in normal subjects in the Cardiovascular Health Study. Neurology 70: 1664-1671. doi: 10.1212/01.wnl.0000306696.82017.66. PubMed: 18401021.

20. Pérez-Grijalba V, Pesini P, Monleón I, Boada M, Tárraga L et al. (2013) Several Direct and Calculated Biomarkers from the Amyloid-beta Pool in Blood are Associated with an Increased Likelihood of Suffering from
Mild Cognitive Impairment. J Alzheimers Dis 36: 211-219. PubMed: 23635404

21. Pesini P, Perez-Grijalba V, Monleon I, Boada M, Tarraga L, et al. (2012) Reliable Measurements of the beta-Amyloid Pool in Blood Could Help in the Early Diagnosis of AD. International journal of Alzheimer's disease 2012: 604141

22. Loring JF, Wen X, Lee JM, Seilhamer J, Somogyi R (2001) A gene expression profile of Alzheimer's disease. DNA Cell Biol 20: 683-695. doi:10.1089/10445490152717541. PubMed: 11788046.

23. Nagasaka $Y$, Dillner $K$, Ebise $H$, Teramoto $R$, Nakagawa $H$ et al. (2005) A unique gene expression signature discriminates familial Alzheimer's disease mutation carriers from their wild-type siblings. Proc Natl Acad Sci U S A 102: 14854-14859. doi:10.1073/pnas.0504178102. PubMed: 16199521.

24. Jin XF, Wu N, Wang L, Li J (2013) Circulating MicroRNAs: A Novel Class of Potential Biomarkers for Diagnosing and Prognosing Central Nervous System Diseases. Cell Mol Neurobiol 33: 601-613. doi: 10.1007/s10571-013-9940-9. PubMed: 23633081.

25. Ray S, Britschgi M, Herbert C, Takeda-Uchimura Y, Boxer A et al. (2007) Classification and prediction of clinical Alzheimer's diagnosis based on plasma signaling proteins. Nat Med 13: 1359-1362. doi: 10.1038/nm1653. PubMed: 17934472

26. Fukumoto H, Tennis M, Locascio JJ, Hyman BT, Growdon JH et al. (2003) Age but not diagnosis is the main predictor of plasma amyloid beta-protein levels. Arch Neurol 60: 958-964. doi:10.1001/archneur. 60.7.958. PubMed: 12873852.

27. Lambert JC, Schraen-Maschke S, Richard F, Fievet N, Rouaud O et al. (2009) Association of plasma amyloid beta with risk of dementia: the prospective Three-City Study. Neurology 73: 847-853. doi:10.1212/ WNL.0b013e3181b78448. PubMed: 19752451.

28. Mayeux R, Honig LS, Tang MX, Manly J, Stern Y et al. (2003) Plasma A[beta]40 and $A[$ beta]42 and Alzheimer's disease: relation to age, mortality, and risk. Neurology 61: 1185-1190. doi:10.1212/01.WNL. 0000091890.32140.8F. PubMed: 14610118

29. Ajmani RS, Metter EJ, Jaykumar R, Ingram DK, Spangler EL et al. (2000) Hemodynamic changes during aging associated with cerebral blood flow and impaired cognitive function. Neurobiol Aging 21: 257-269. doi:10.1016/S0197-4580(00)00118-4. PubMed: 10867210.

30. Abdullah L, Paris D, Luis C, Quadros A, Parrish J et al. (2007) The influence of diagnosis, intra- and inter-person variability on serum and plasma Abeta levels. Neurosci Lett 428: 53-58. doi:10.1016/j.neulet. 2007.09.058. PubMed: 17964720

31. Crawford F, Soto C, Suo Z, Fang C, Parker T et al. (1998) Alzheimer's beta-amyloid vasoactivity: identification of a novel beta-amyloid conformational intermediate. FEBS Lett 436: 445-448. doi:10.1016/ S0014-5793(98)01170-3. PubMed: 9801166.

32. Crawford F, Suo Z, Fang C, Mullan M (1998) Characteristics of the in vitro vasoactivity of beta-amyloid peptides. Exp Neurol 150: 159-168. doi:10.1006/exnr.1997.6743. PubMed: 9514824

33. Sawmiller DR, Nguyen HT, Markov O, Chen M (2012) High-energy compounds promote physiological processing of Alzheimer's amyloidbeta precursor protein and boost cell survival in culture. J Neurochem 123: 525-531. doi:10.1111/j.1471-4159.2012.07923.x. PubMed: 22906069.

34. Ishii T, Haga S (1975) Identification of components of immunoglobulins in senile plaques by means of fluorescent antibody technique. Acta Neuropathol 32: 157-162. doi:10.1007/BF00689569. PubMed: 809980.

35. Niranjan R (2013) Molecular Basis of Etiological Implications in Alzheimer's Disease: Focus on Neuroinflammation. Mol Neurobiol, 48: 412-28. PubMed: 23420079.

36. Alegret M, Espinosa A, Vinyes-Junqué G, Valero S, Hernández I et al. (2012) Normative data of a brief neuropsychological battery for Spanish individuals older than 49. J Clin Exp Neuropsychol 34: 209-219. doi: 10.1080/13803395.2011.630652. PubMed: 22149440.

37. Petersen RC, Smith GE, Waring SC, Ivnik RJ, Tangalos EG et al. (1999) Mild cognitive impairment: clinical characterization and outcome. Arch Neurol 56: 303-308. doi:10.1001/archneur.56.3.303. PubMed: 10190820

38. Petersen RC, Morris JC (2005) Mild cognitive impairment as a clinical entity and treatment target. Arch Neurol 62: 1160-1163; discussion: 10.1001/archneur.62.7.1160. PubMed: 16009779.

39. Dubois B, Feldman HH, Jacova C, Dekosky ST, Barberger-Gateau P et al. (2007) Research criteria for the diagnosis of Alzheimer's disease: revising the NINCDS-ADRDA criteria. Lancet Neurol 6: 734-746. doi: 10.1016/S1474-4422(07)70178-3. PubMed: 17616482.

40. McKhann G, Drachman D, Folstein M, Katzman R, Price D et al. (1984) Clinical diagnosis of Alzheimer's disease: report of the NINCDS- 
ADRDA Work Group under the auspices of Department of Health and Human Services Task Force on Alzheimer's Disease. Neurology 34: 939-944. doi:10.1212/WNL.34.7.939. PubMed: 6610841.

41. Blesa R, Pujol M, Aguilar M, Santacruz P, Bertran-Serra I et al. (2001) Clinical validity of the 'mini-mental state' for Spanish speaking communities. Neuropsychologia 39: 1150-1157. doi:10.1016/ S0028-3932(01)00055-0. PubMed: 11527552.

42. R_Core_Team (2013) R: A language and environment for statistical computing. Vienna, Austria: R Foundation for Statistical Computing. 